\title{
Exploratory analysis of the platelet-to-lymphocyte ratio prognostic value in the adjuvant renal cell cancer setting
}

\author{
Anup Patel*,1(i), Alain Ravaud², Robert J Motzer ${ }^{3}$, Allan J Pantuck ${ }^{4}$, Michael Staehler $^{5}$, \\ Bernard Escudier ${ }^{6}$, Jean-François Martini ${ }^{7}$, Mariajose Lechuga ${ }^{8}$, Xun Lin ${ }^{7}$ \& Daniel J George ${ }^{9}$ \\ ${ }^{1}$ Private Residence, London, UK \\ ${ }^{2}$ Bordeaux University Hospital, 33000 Bordeaux, France \\ ${ }^{3}$ Memorial Sloan Kettering Cancer Center, New York, NY 10065, USA \\ ${ }^{4}$ UCLA David Geffen School of Medicine, Los Angeles, CA 90094, USA \\ ${ }^{5}$ University Hospital of Munich, 80336 Munich, Germany \\ ${ }^{6}$ Institut Gustave Roussy, 94800 Villejuif, France \\ ${ }^{7}$ Pfizer Inc., La Jolla, CA 92121, USA \\ ${ }^{8}$ Pfizer S.r.L, Via Anna Maria Mozzoni, 12, 20152 Milan, Italy \\ ${ }^{9}$ Duke Cancer Institute, Durham, NC 27710, USA \\ *Author for correspondence: anuppatel666@gmail.com
}

Aim: To examine the prognostic value of the platelet-to-lymphocyte ratio (PLR) in the adjuvant renal cell carcinoma setting. Materials \& methods: Patients received adjuvant sunitinib (50 mg/day; 4 weeks on/2 weeks off) or placebo. The primary end point was disease-free survival (DFS). Results: In 609 patients, DFS was similar for baseline PLR $<140$ versus $\geq 140$ overall (median: 6.4 vs 5.9 years; hazard ratio: 0.9; $95 \%$ $\mathrm{Cl}$ : 0.7-1.2). $A \geq 25 \%$ decrease in PLR at week 4 overall was associated with longer DFS versus no change (hazard ratio: 0.8; $95 \% \mathrm{Cl}$ : 0.6-1.0). Conclusion: Baseline PLR was not prognostic for DFS with adjuvant sunitinib treatment in patients with renal cell carcinoma.

Clinical Trials Registration: NCT00375674 (ClinicalTrials.gov)

First draft submitted: 26 June 2020; Accepted for publication: 16 September 2020; Published online: 8 October 2020

Keywords: adjuvant $\bullet$ lymphocyte $\bullet$ platelet $\bullet$ prognostic $\bullet$ renal cell carcinoma $\bullet$ sunitinib

Cancer cells release many chemical mediators that directly and indirectly modulate inflammation and immune processes. This includes platelet activation, which may promote cancer progression through multiple mechanisms, including enhanced angiogenesis, cell proliferation, inflammation, immune surveillance evasion, and by enhancing invasive potential and extravasation of cancer cells $[1,2]$. The platelet-to-lymphocyte ratio (PLR) is a surrogate for systemic inflammation [3]. PLR has shown promise as a prognostic marker of clinical outcomes for a variety of cancers, including localized and metastatic renal cell carcinoma (RCC) [4-6], where elevated PLR was prognostic for worse outcomes. Patients with locoregional RCC with a high risk of recurrence represent approximately 15\% of the overall RCC population [7], and over a 5-year period, their recurrence rate is approximately 60\% [7]. This indicates a clear unmet need for effective adjuvant therapy. In the S-TRAC trial, adjuvant sunitinib significantly improved disease-free survival (DFS) versus placebo (median: 6.8 vs 5.6 years; hazard ratio [HR]: 0.76; 95\% CI: 0.59-0.98; $p=0.03$ [8]. Using data from S-TRAC to retrospectively identify biomarkers associated with greater clinical benefit from adjuvant sunitinib therapy could inform future clinical studies. This exploratory analysis of S-TRAC evaluated any possible prognostic and/or predictive value of PLR in patients with locoregional clear-cell RCC at high risk of recurrence post nephrectomy.

\section{Materials \& methods}

S-TRAC was a double-blind, randomized, multicenter Phase III trial that randomized 615 patients in a 1:1 ratio to receive either adjuvant sunitinib $(50 \mathrm{mg} /$ day) or placebo on a 4-weeks-on/2-weeks-off schedule for 1 year

Future $\because \cdots$ Medicine 


\begin{tabular}{|c|c|c|c|}
\hline Characteristic & $\begin{array}{l}\text { PLR }<140 \\
(n=325)\end{array}$ & $\begin{array}{l}\text { PLR } \geq 140 \\
(n=284)\end{array}$ & $\begin{array}{l}\text { Total } \\
(n=609)\end{array}$ \\
\hline Age, median (range), years & $58.0(27-83)$ & $56.0(21-82)$ & $58.0(21-83)$ \\
\hline \multicolumn{4}{|l|}{ Sex, $\mathrm{n}(\%)$} \\
\hline- Male & $247(76.0)$ & $199(70.1)$ & $446(73.2)$ \\
\hline - Female & $78(24.0)$ & $85(29.9)$ & $163(26.8)$ \\
\hline \multicolumn{4}{|l|}{ ECOG PS, n (\%) } \\
\hline-0 & $240(73.8)$ & $204(71.8)$ & $444(72.9)$ \\
\hline-1 & $85(26.2)$ & $76(26.8)$ & $161(26.4)$ \\
\hline-2 & 0 & $1(0.4)$ & $1(0.2)$ \\
\hline - Not reported & 0 & $3(1.1)$ & $3(0.5)$ \\
\hline \multicolumn{4}{|l|}{ UISS high-risk group, $\mathrm{n}(\%)$} \\
\hline - T3 N0 or NX, M0, Fuhrman's grade $\geq 2$, ECOG PS $\geq 1$ & $178(54.8)$ & $149(52.5)$ & $327(53.7)$ \\
\hline $\begin{array}{l}\text {-T3 N0 or NX, M0, any Fuhrman's grade, ECOG PS } 0 \text {, or T3 N0 or } \\
\text { NX, MO, Fuhrman's grade }=1, \text { ECOG PS } \geq 1\end{array}$ & $120(36.9)$ & $106(37.3)$ & $226(37.1)$ \\
\hline - T4 N0 or NX, MO, any Fuhrman's grade, any ECOG PS & $2(0.6)$ & $6(2.1)$ & $8(1.3)$ \\
\hline - Any T, N1-2, M0, any Fuhrman's grade, any ECOG PS & $25(7.7)$ & $23(8.1)$ & $48(7.9)$ \\
\hline \multicolumn{4}{|l|}{ Fuhrman's grade, n (\%) } \\
\hline-1 & $11(3.4)$ & $8(2.8)$ & $19(3.1)$ \\
\hline-2 & $116(35.7)$ & $91(32.0)$ & $207(34.0)$ \\
\hline-3 & $146(44.9)$ & $131(46.1)$ & $277(45.5)$ \\
\hline-4 & $52(16.0)$ & $52(18.3)$ & $104(17.1)$ \\
\hline - Not reported & 0 & $2(0.7)$ & $2(0.3)$ \\
\hline
\end{tabular}

(ClinicalTrials.gov: NCT00375674) [8]. The trial was conducted in compliance with the International Conference on Harmonisation Good Clinical Practice guidelines, with the ethical principles originating in or derived from the Declaration of Helsinki, and in accordance with applicable local regulatory requirements and laws. The independent review board or ethics committee at each participating center approved the trial, and all patients provided written informed consent.

The primary end point was DFS, determined by blinded independent central review, defined as the time from randomization to recurrence or occurrence of metastasis, a secondary primary malignancy, or death, whichever occurred first. High-risk patients were defined as those patients with $\geq \mathrm{pT} 3$ and/or $\mathrm{N}^{+}$tumors based on the UCLA Integrated Staging System (UISS). The baseline measure of PLR was determined postsurgery and no earlier than 2 weeks before randomization and initiation of treatment. Receiver-operating characteristic (ROC) plots were applied to baseline PLR data to identify optimal PLR cutoff values. Kaplan-Meier analyses were performed on baseline PLR values and the change in PLR at the end of the first dosing cycle (week 4) to assess their association with DFS. Week 4 was selected because hematology and laboratory chemistry tests were conducted on day 28 at the time when patients had completed their first treatment cycle. Statistical comparison of baseline PLR data with DFS was performed using a two-sided unstratified log-rank test. Statistical comparison of the change from the baseline PLR value to the week 4 value with DFS was based on a two-sided log-rank test stratified by the UISS high-risk group. Significance was set at $\mathrm{p}<0.05$ for all statistical tests.

\section{Results}

In total, data from 609 patients were available for analysis. ROC analysis identified an optimal cutoff value for baseline PLR of 140. As a result, 325 patients (53.4\%) had PLR $<140$ and 284 (46.6\%) had PLR $\geq 140$ (Figure 1 A). Demographic and baseline characteristics of the total patient population and by PLR cutoff $(<140$ vs $\geq 140)$ are shown in Table 1. Characteristics were similar irrespective of PLR category. 
(A)

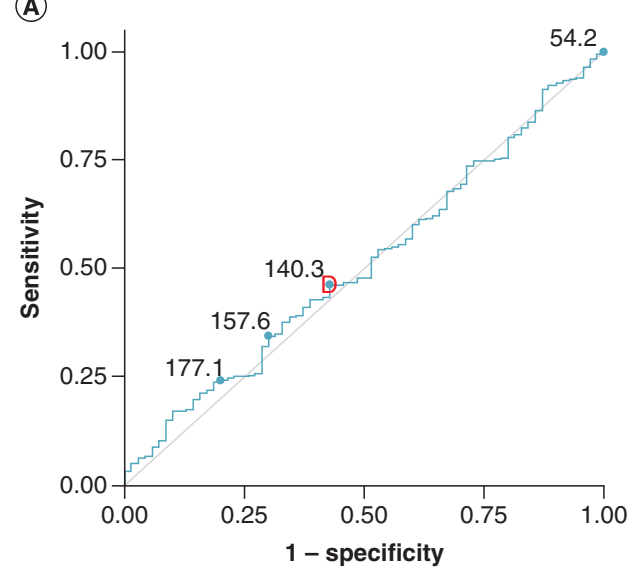

(B)
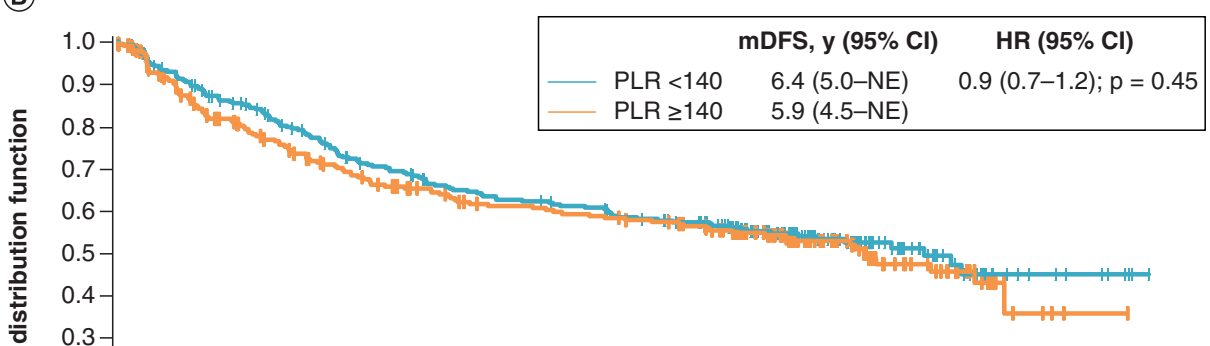

㟔 0

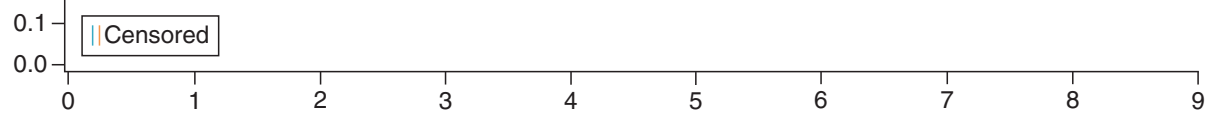

No. at risk Disease-free survival (years)

$\begin{array}{lllllllllll}<140 & 325 & 246 & 194 & 170 & 153 & 122 & 47 & 11 & 4 & 0 \\ \geq 140 & 284 & 195 & 156 & 130 & 123 & 96 & 41 & 8 & 1 & 0\end{array}$

(C)

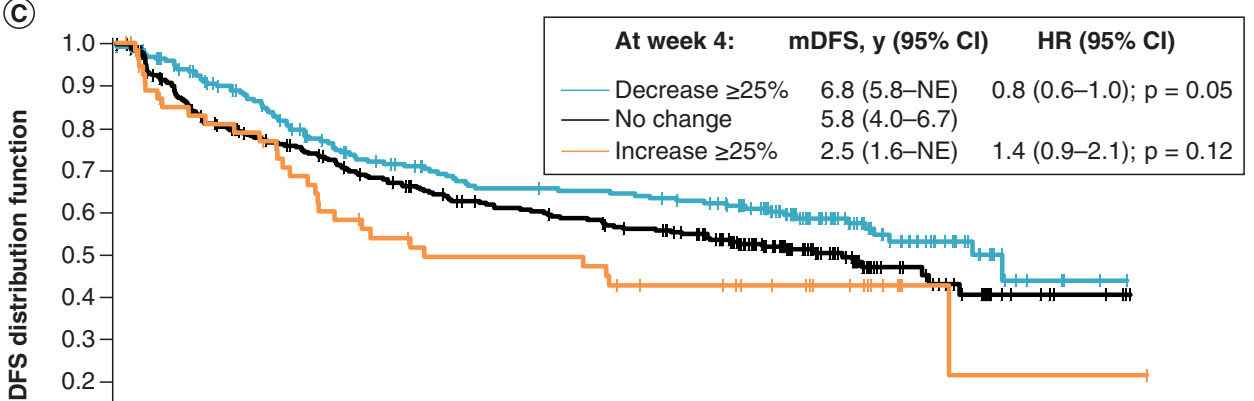

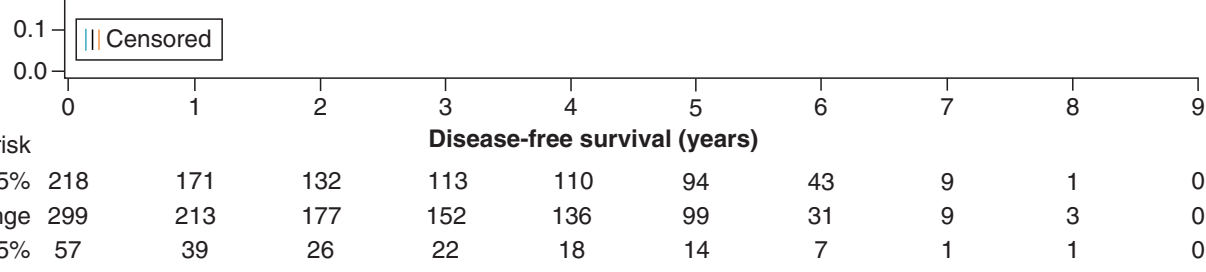

Figure 1. Optimal cutoff for baseline platelet-to-lymphocyte ratio and treatment outcomes by baseline platelet-to-lymphocyte ratio. (A) ROC analysis of baseline PLR. (B) DFS by baseline PLR. (C) Change in PLR from baseline at week 4 . In (A), the red D-symbol refers to the optimal cutoff point. Data in (B) and (C) are from the overall patient population. (B) Data are for PLR of $<140$ versus $\geq 140$. $p$-values were two-sided and based on an unstratified log-rank test. (C) Data are for $\geq 25 \%$ decrease in PLR versus no change versus $\geq 25 \%$ increase in PLR. Patients without PLR values at baseline or week 4 were excluded. p-values were two-sided and based on a log-rank test stratified by the UISS high-risk group.

DFS: Disease-free survival; HR: Hazard ratio; mDFS: Median DFS; NE: Not estimable; PLR: Platelet-to-lymphocyte ratio; ROC: Receiver-operating characteristic; UISS: UCLA Integrated Staging System. 
In the overall population, there was no difference in DFS for baseline PLR $<140$ versus $\geq 140$ (median: 6.4 vs 5.9 years; HR: $0.9 ; 95 \%$ CI: $0.7-1.2 ; \mathrm{p}=0.45$ ) (Figure $1 \mathrm{~B}$ ). There was also no difference in DFS for baseline PLR $<140$ versus $\geq 140$ in the sunitinib (median: not reached vs 6.8 years; HR: $0.8 ; 95 \%$ CI: $0.6-1.2 ; \mathrm{p}=0.33$ ) or placebo (median: 5.6 vs 5.3 years; HR: 1.0; 95\% CI: $0.7-1.4 ; \mathrm{p}=0.87$ ) treatment arms. There was a trend toward longer DFS for sunitinib versus placebo for baseline PLR $<140$ (median: not reached vs 5.6 years; HR: 0.7; 95\% CI: $0.5-1.0 ; \mathrm{p}=0.06$ ). However, in patients with baseline PLR $\geq 140$, DFS was similar for sunitinib versus placebo (median: 6.8 vs 5.3 years; HR: 0.8 ; $95 \%$ CI: $0.6-1.2 ; \mathrm{p}=0.27$ ).

Change in PLR from baseline at week 4 (i.e., at the end of the first treatment cycle) was determined and its association with DFS examined. In the sunitinib treatment arm $(\mathrm{n}=281), 175(62.3 \%)$ patients had $\mathrm{a} \geq 25 \%$ decrease in PLR from baseline at week 4, 74 (26.3\%) had no change, and $32(11.4 \%)$ had a $\geq 25 \%$ increase. By comparison, in the placebo treatment arm $(\mathrm{n}=293), 43(14.7 \%)$ patients had $\mathrm{a} \geq 25 \%$ decrease in PLR from baseline at week 4, $225(76.8 \%)$ had no change and $25(8.5 \%)$ had a $\geq 25 \%$ increase. The distributions of the change in PLR at week 4 were statistically significantly different $(\mathrm{p}<0.0001)$ between sunitinib and placebo. In the overall population, $\mathrm{a} \geq 25 \%$ decrease in PLR at week 4 was associated with longer DFS versus no change (median: 6.8 vs 5.8 years; HR: $0.8 ; 95 \%$ CI: $0.6-1.0 ; \mathrm{p}=0.05$ ) (Figure $1 \mathrm{C}$ ), but it was not statistically significantly different in those with $\mathrm{a} \geq 25 \%$ increase versus no change (median: 2.5 vs 5.8 years; HR: $1.4 ; 95 \%$ CI: $0.9-2.1 ; \mathrm{p}=0.12$ ). In the sunitinib arm, there was no difference in DFS in patients with $\mathrm{a} \geq 25 \%$ decrease in PLR at week 4 versus no change (median: 7.1 vs 5.9 years; HR: $0.8 ; 95 \%$ CI: 0.5-1.2; $\mathrm{p}=0.27$ ). Similarly, there was no difference in DFS in patients with $\mathrm{a} \geq 25 \%$ decrease in PLR at week 4 versus no change in the placebo arm (median: 5.8 vs 5.3 years; HR: $1.1 ; 95 \%$ CI: $0.7-1.7 ; \mathrm{p}=0.76$ ). At week 4 , patients with a $\geq 25 \%$ decrease in PLR received a numerically greater median total cumulative dose $(9856.3 \mathrm{mg})$ compared with patients with no change $(9418.8 \mathrm{mg})$ and those with $\mathrm{a} \geq 25 \%$ increase $(6424.0 \mathrm{mg})$.

\section{Discussion}

In this exploratory analysis of data from the S-TRAC study of adjuvant sunitinib versus placebo in patients with locoregional clear-cell RCC at high risk of recurrence post nephrectomy, baseline PLR was not found to be a useful predictor of DFS. Overall, the change in PLR from baseline to the end of the first treatment cycle (i.e., week 4) appeared to be prognostic for outcome $(\mathrm{p}=0.05)$. Change in PLR to the end of the first treatment cycle may be a novel, easily applicable and inexpensive prognostic factor to assess. Indeed, incorporating PLR instead of platelet count improves the prognostic accuracy of the International Metastatic Renal Cell Carcinoma Database Consortium model [9]. Moreover, significantly more patients in the sunitinib arm reported a $\geq 25 \%$ decrease in PLR compared with placebo. However, in neither the sunitinib nor placebo arms, there was a significant difference in DFS between those with $\mathrm{a} \geq 25 \%$ decrease in PLR and no change ( $\mathrm{p}=0.27$ and 0.76 , respectively). It is therefore possible that the apparent prognostic ability of change in PLR to week 4 is simply a proxy measure for the greater reduction in platelets with sunitinib treatment.

An additional finding from this analysis was that patients who had $\mathrm{a} \geq 25 \%$ decrease in PLR at week 4 received a larger cumulative dose of sunitinib compared with those patients who experienced no change in PLR or $a \geq 25 \%$ increase. The change in PLR at the end of the first treatment cycle could therefore be used to identify patients most likely to tolerate and maintain sunitinib dose, and thereby continue to accrue potential benefit from treatment, while needless treatment-related toxicity risk could be reduced in those unlikely to accrue potential benefit.

The lack of prognostic value of baseline PLR in the current analysis is in contrast to previous studies in advanced RCC where elevated PLR was prognostic for shorter progression-free survival, overall survival, recurrence-free survival and cancer-specific survival, compared with lower PLR levels [4-6,10-15]. This may reflect intrinsic study differences in patient inclusion criteria, differences in type of inflammation (inflammation associated with tumor volume vs postsurgical inflammation), treatment setting (nonadjuvant vs adjuvant), the use of DFS as the treatment end point and study power. Moreover, postoperative PLR values may have been affected by differing time intervals after surgery at the time of study recruitment, and there may have been confounding blood transfusions in a very small number of patients, since the advent of laparoscopic radical nephrectomy in this patient population, which were not documented on case report forms, further contributed to a negative result. Reasons for the discrepancies between this analysis and other studies warrant further exploration. For instance, the prognostic value of PLR in other studies of adjuvant treatment for RCC with similar risk patient populations as in S-TRAC, such as the ASSURE [16] and PROTECT [17] trials, could be examined. 
In the S-TRAC trial, the day of nephrectomy was considered as 'day 0,' therefore preoperative patient data, such as clinical laboratory blood tests and including platelet and lymphocyte levels, were not captured. Given that the trial was in the adjuvant treatment setting, it would be informative to further analyze whether prenephrectomy PLR values were prognostic for outcomes. However, this was not possible in this post hoc analysis of the existing S-TRAC dataset. If other datasets in the adjuvant treatment setting, such as for ASSURE or PROTECT [16,17], did capture prenephrectomy patient data including laboratory values, such an analysis could be conducted.

A separate exploratory analysis of data from S-TRAC examined the prognostic capability of the neutrophil-tolymphocyte ratio (NLR) on DFS [18]. Cutoff values of $<3$ and $\geq 3$ for baseline NLR were identified using ROC analysis. Overall, median DFS was shorter in the NLR $<3$ group versus the NLR $\geq 3$ group (HR: 1.4; $\mathrm{p}=0.04$ ), and patients in the NLR $<3$ group who received sunitinib had longer median DFS than those who received placebo (HR: 0.7; $\mathrm{p}=0.02$ ). $\mathrm{A} \geq 25 \%$ decrease in NLR at week 4 was associated with longer DFS versus no change (HR: $0.7 ; \mathrm{p}=0.01)$, and more patients in the sunitinib group had $\mathrm{a} \geq 25 \%$ decrease at week 4 versus placebo $(71.2 \mathrm{vs}$ $17.4 \%)$. Baseline NLR level and change in NLR after the first adjuvant sunitinib treatment cycle appeared to be prognostic for DFS in S-TRAC.

\section{Conclusion}

In summary, this exploratory analysis showed that baseline PLR was not prognostic for DFS in patients with locoregional clear-cell RCC at high risk of recurrence post nephrectomy treated with adjuvant sunitinib, possibly due to a lack of statistical power and postoperative hemodynamic effects in this study population, where baseline prenephrectomy data were not collected.

\section{Summary points}

- Elevated platelet-to-lymphocyte ratio (PLR) is a prognostic marker of worse clinical outcomes in advanced renal cell carcinoma (RCC) but has not been tested in the earlier adjuvant treatment setting.

- In the S-TRAC trial, adjuvant sunitinib significantly improved disease-free survival (DFS) versus placebo in patients with locoregional clear-cell RCC at high risk of recurrence post nephrectomy.

- This exploratory analysis examined the prognostic value of PLR using data from the S-TRAC study.

- Receiver-operating characteristic analysis identified an optimal cutoff value for baseline PLR of $140(53.4 \%$ had PLR $<140$, and $46.6 \%$ had PLR $\geq 140$ ).

- In 609 patients, DFS was similar for baseline PLR $<140$ versus $\geq 140$ overall (median: 6.4 vs 5.9 years; hazard ratio [HR]: $0.9 ; 95 \% \mathrm{Cl}: 0.7-1.2$ ), and in each treatment arm (sunitinib: HR: 0.8; 95\% Cl: 0.6-1.2; placebo: HR: $1.0 ; 95 \%$ Cl: 0.7-1.4).

- Proportionally, more sunitinib-treated patients had a $\geq 25 \%$ PLR decrease at week 4 versus placebo (62.3 vs $14.7 \%)$.

- $\mathrm{A} \geq 25 \%$ decrease in PLR at week 4 overall was associated with longer DFS versus no change (HR: $0.8 ; 95 \% \mathrm{Cl}$ : $0.6-1.0$ ), but there was no difference in the sunitinib arm (HR: $0.8 ; 95 \% \mathrm{Cl}: 0.5-1.2$ ).

- In conclusion, baseline PLR did not demonstrate prognostic value for DFS in the adjuvant sunitinib treatment setting in patients with RCC at high risk of recurrence post nephrectomy.

\section{Author contributions}

A Patel, A Ravaud, RJ Motzer, AJ Pantuck, M Staehler, B Escudier, J-F Martini, M Lechuga, X Lin and DJ George were responsible for the conception and design. A Patel, A Ravaud, RJ Motzer, AJ Pantuck, M Staehler, B Escudier and DJ George were responsible for the acquisition of data. J-F Martini, M Lechuga and X Lin analyzed the data. A Patel, A Ravaud, RJ Motzer, AJ Pantuck, M Staehler, B Escudier, J-F Martini, M Lechuga, X Lin and DJ George were responsible for drafting and revision of the manuscript.

Financial and competing interests disclosure

This study was sponsored by Pfizer. A Patel has received consulting fees from and served on a steering committee for Pfizer. A Ravaud has received fees for serving on advisory boards from Pfizer, Novartis, GlaxoSmithKline, Bristol-Myers Squibb, Merck GA, Merck Sharp \& Dohme, Ipsen and Roche; lecture fees from Pfizer, Novartis, Ipsen and GlaxoSmithKline; travel support from Pfizer, Novartis, GlaxoSmithKline, Bristol-Myers Squibb, Ipsen, Merck GA and Merck Sharp \& Dohme; and grant support from Pfizer and Novartis. RJ Motzer has received commercial research grants from Bristol-Myers Squibb, Pfizer, Novartis, Genentech/Roche and Eisai; and is a consultant and/or advisory board member for Genentech, Pfizer, Merck, Novartis, Incyte, Roche, Eisai, Exelixis and Lilly. AJ Pantuck has received consulting fees from and served on a steering committee for Pfizer. M Staehler has received grant 
support and fees for serving on advisory boards from Pfizer, GlaxoSmithKline, Novartis, Bayer, Exelixis and Roche; and consulting fees, honoraria and travel support from Pfizer, GlaxoSmithKline, Novartis, Bayer and Roche. B Escudier has received fees for serving on advisory boards from Pfizer, Novartis, Bristol-Myers Squibb, Exelixis and Roche; and lecture fees from Pfizer and Novartis. J-F Martini, M Lechuga and X Lin are employees of Pfizer and have stock or stock options with Pfizer. DJ George has received consulting fees from Sanofi, Exelixis, Bayer, Merck, Janssen, Pfizer, Innocrin Pharmaceuticals and Bristol-Myers Squibb; honoraria from Sanofi, Exelixis and Bayer; and grants from Genentech/Roche, Novartis, Astellas, Celldex Therapeutics, Acerta Pharma, Exelixis, Janssen, Pfizer, Innocrin Pharmaceuticals and Bristol-Myers Squibb. Patients treated at the Memorial Sloan Kettering Cancer Center were supported in part by Memorial Sloan Kettering Cancer Center Support Grant/Core Grant P30 CA008748. The authors have no other relevant affiliations or financial involvement with any organization or entity with a financial interest in or financial conflict with the subject matter or materials discussed in the manuscript apart from those disclosed.

Medical writing support was provided by David Cope, PhD, of Engage Scientific Solutions, and funded by Pfizer.

Ethical conduct of research

The original S-TRAC trial was conducted in compliance with the International Conference on Harmonisation Good Clinical Practice guidelines, with the ethical principles originating in or derived from the Declaration of Helsinki, and in accordance with applicable local regulatory requirements and laws. The independent review board or ethics committee at each participating center approved the trial, and all patients provided written informed consent.

\section{Data sharing statement}

Upon request, and subject to certain criteria, conditions and exceptions (https://www.pfizer.com/science/clinical-trials/trial-dataand-results for more information), Pfizer will provide access to individual deidentified participant data from Pfizer-sponsored global interventional clinical studies conducted for medicines, vaccines and medical devices for indications that have been approved in the USA and/or EU, or in programs that have been terminated (i.e., development for all indications has been discontinued). Pfizer will also consider requests for the protocol, data dictionary and statistical analysis plan. Data may be requested from Pfizer trials 24 months after study completion. The deidentified participant data will be made available to researchers whose proposals meet the research criteria and other conditions, and for which an exception does not apply, via a secure portal. To gain access, data requestors must enter into a data access agreement with Pfizer.

\section{Open access}

This work is licensed under the Attribution-NonCommercial-NoDerivatives 4.0 Unported License. To view a copy of this license, visit http://creativecommons.org/licenses/by-nc-nd/4.0/

\section{References}

Papers of special note have been highlighted as: $\bullet$ of interest; $\bullet \bullet$ of considerable interest

1. Li N. Platelets in cancer metastasis: to help the "villain" to do evil. Int. J. Cancer 138(9), 2078-2087 (2016).

- Overview of the putative roles, platelets may play in multiple processes involved in cancer metastasis.

2. Olsson AK, Cedervall J. The pro-inflammatory role of platelets in cancer. Platelets 29(6), 569-573 (2018).

3. Kumarasamy C, Sabarimurugan S, Madurantakam RM et al. Prognostic significance of blood inflammatory biomarkers NLR, PLR, and LMR in cancer - a protocol for systematic review and meta-analysis. Medicine (Baltimore) 98(24), e14834 (2019).

4. Albisinni S, Pretot D, Al Hajj Obeid W et al. The impact of neutrophil-to-lymphocyte, platelet-to-lymphocyte and haemoglobin-to-platelet ratio on localised renal cell carcinoma oncologic outcomes. Prog. Urol. 29(8-9), 423-431 (2019).

5. Semeniuk-Wojtas A, Lubas A, Stec R, Syrylo T, Niemczyk S, Szczylik C. Neutrophil-to-lymphocyte ratio, platelet-to-lymphocyte ratio, and $\mathrm{C}$-reactive protein as new and simple prognostic factors in patients with metastatic renal cell cancer treated with tyrosine kinase inhibitors: a systemic review and meta-analysis. Clin. Genitourin. Cancer. 16(3), e685-e693 (2018).

- Systematic review and meta-analysis of inflammatory biomarkers, including the platelet-to-lymphocyte ratio (PLR), and their association with tyrosine kinase inhibitor treatment outcomes in patients with metastatic renal cell carcinoma (RCC). In the meta-analysis, four studies included data on PLR. Elevated PLR was associated with worse overall survival (hazard ratio [HR]: 14.7; 95\% CI: 11.1-19.6; $\mathrm{p}<\mathbf{0 . 0 0 1}$ ) and progression-free survival (HR: 7.0; 95\% CI: 5.0-9.6; $\mathrm{p}<0.001$ ).

6. Wang Z, Peng S, Wang A et al. Platelet-lymphocyte ratio acts as an independent predictor of prognosis in patients with renal cell carcinoma. Clin. Chim. Acta 480, 166-172 (2018).

- Systematic review and meta-analysis examining PLR as a prognostic factor in RCC. Data from 16 studies (6223 patients) were included and showed that elevated PLR was associated with with worse overall survival (HR: 1.76; 95\% CI: 1.41-2.19; p < 0.001), progression-free survival (HR: 2.81; 95\% CI: 1.40-5.63; $p=0.004$ ) and recurrence-free survival (HR: 2.64; 95\% CI: 1.35-5.14; $p=0.004)$. 
7. Lam JS, Shvarts O, Leppert JT, Pantuck AJ, Figlin RA, Belldegrun AS. Postoperative surveillance protocol for patients with localized and locally advanced renal cell carcinoma based on a validated prognostic nomogram and risk group stratification system. J. Urol. 174(2), 466-472 (2005).

8. Ravaud A, Motzer RJ, Pandha HS et al. Adjuvant sunitinib in high-risk renal-cell carcinoma after nephrectomy. N. Engl. J. Med. 375(23), 2246-2254 (2016).

-• Randomized, placebo-controlled, double-blind, Phase III trial (S-TRAC) of adjuvant sunitinib in patients with high-risk RCC after nephrectomy. Disease-free survival (DFS) was significantly longer in the sunitinib group versus placebo (median: 6.8 vs 5.6 years; HR: 0.76; 95\% CI: 0.59-0.98; $p=0.03$ ) at a cost of higher incidences of Grade 3 or 4 adverse events.

9. Chrom P, Stec R, Bodnar L, Szczylik C. Incorporating neutrophil-to-lymphocyte ratio and platelet-to-lymphocyte ratio in place of neutrophil count and platelet count improves prognostic accuracy of the International Metastatic Renal Cell Carcinoma Database Consortium Model. Cancer Res. Treat. 50(1), 103-110 (2018).

-• Retrospective analysis testing if replacing neutrophil count and platelet count with neutrophil-to-lymphocyte ratio (NLR) and PLR in the International Metastatic Renal Cell Carcinoma Database Consortium (IMDC) model improved prognostic accuracy. Using an NLR value of 3.6 and PLR value of 157, all measures of prognostic accuracy were improved in the modified-IMDC model, compared with the standard IMDC model.

10. Huszno J, Kolosza Z, Mrochem-Kwarciak J, Rutkowski T, Skladowski K. The role of neutrophil-lymphocyte ratio, platelet-lymphocyte ratio, and platelets in the prognosis of metastatic renal cell carcinoma. Oncology 97, 7-17 (2019).

11. Wang X, Su S, Guo Y. The clinical use of the platelet to lymphocyte ratio and lymphocyte to monocyte ratio as prognostic factors in renal cell carcinoma: a systematic review and meta-analysis. Oncotarget 8(48), 84506-84514 (2017).

12. Hu H, Yao X, Xie X et al. Prognostic value of preoperative NLR, dNLR, PLR and CRP in surgical renal cell carcinoma patients. World J. Urol. 35(2), 261-270 (2017).

13. Kim TW, Lee JH, Shim KH et al. Prognostic significance of preoperative and follow-up neutrophil-to-lymphocyte ratio and platelet-to-lymphocyte ratio in patients with non-metastatic clear cell renal cell carcinoma. Investig. Clin. Urol. 60(1), 14-20 (2019).

14. Park TJ, Cho YH, Chung HS et al. Prognostic significance of platelet-lymphocyte ratio in patients receiving first-line tyrosine kinase inhibitors for metastatic renal cell cancer. Springerplus 5(1), 1889 (2016).

15. Gunduz S, Mutlu H, Tural D et al. Platelet to lymphocyte ratio as a new prognostic for patients with metastatic renal cell cancer. Asia Pac. J. Clin. Oncol. 11(4), 288-292 (2015).

16. Haas NB, Manola J, Uzzo RG et al. Adjuvant sunitinib or sorafenib for high-risk, non-metastatic renal-cell carcinoma (ECOG-ACRIN E2805): a double-blind, placebo-controlled, randomised, Phase III trial. Lancet 387(10032), 2008-2016 (2016).

17. Motzer RJ, Haas NB, Donskov F et al. Randomized Phase III trial of adjuvant pazopanib versus placebo after nephrectomy in patients with localized or locally advanced renal cell carcinoma. J. Clin. Oncol. 35(35), 3916-3923 (2017).

18. Patel A, Ravaud A, Motzer RJ et al. Neutrophil-to-lymphocyte ratio as a prognostic factor of disease-free survival in post-nephrectomy high-risk loco-regional renal cell carcinoma: analysis of the S-TRAC trial. Clin. Cancer Res. 26(18), 4863-4868 (2020).

-• Post hoc exploratory analysis testing the prognostic value of the NLR for adjuvant sunitinib-treatment outcomes in the S-TRAC trial. Using an NLR cutoff of three, patients with NLR $<3$ had longer DFS versus placebo (median: 7.1 vs 4.7 years; HR: 0.71; $p$ $=0.02$ ). $A \geq 25 \%$ decrease in NLR at week 4 was associated with longer DFS versus no change (median: 6.8 vs 5.3 years; HR: $0.71 ; p=0.01$ ). 
